\title{
Resilience in social-ecological systems: identifying stable and unstable equilibria with agent-based models
}

\author{
$\underline{\text { Maarten J. van Strien }}^{1}$, Sibyl H. Huber ${ }^{1,2}, \underline{\text { John M. Anderies }}^{3}$ and Adrienne Grêt-Regamey $^{1}$
}

\begin{abstract}
To determine the resilience of complex social-ecological systems (SESs) it is necessary to have a thorough understanding of the system behavior under changing political, economic, and environmental conditions (i.e., external system stressors). Such behavior can be predicted if one knows the stable and unstable equilibrium states in a system and how these equilibria react to changes in the system stressors. The state of the system rapidly or gradually changes either toward (i.e., stable equilibrium) or away from (i.e., unstable equilibrium) an equilibrium. However, the equilibrium states in a SES are often unknown and difficult to identify in real systems. In contrast, agent-based SES models can potentially be used to determine equilibria states, but are rarely used for this purpose. We developed a generic approach to identify stable and unstable equilibria states with agent-based SES models. We used an agent-based SES model to simulate land-use change in an alpine mountain region in the Canton of Valais, Switzerland. By iteratively running this model for different input settings, we were able to identify equilibria in intensive and extensive agriculture. We also assessed the sensitivity of these equilibria to changes in external system stressors. With support-vector machine classifications, we created bifurcation diagrams in which the stable and unstable equilibria as a function of the values of a system stressor were depicted. The external stressors had a strong influence on the equilibrium states. We also found that a minimum amount of direct payments was necessary for agricultural extensification to take place. Our approach does not only provide valuable insights into the resilience of our case-study region to changing conditions, but can also be applied to other (agent-based) SES models to present important model results in a condensed and understandable format.
\end{abstract}

Key Words: agricultural land use; bifurcation diagram; land-use change; regime shift

\section{INTRODUCTION}

Changing environmental, ecological, political, and socioeconomic conditions can have far-reaching consequences for the functioning of ecological, social, and coupled social-ecological systems (SESs; Walker et al. 2004, Folke 2006). Empirical studies on ecosystems have shown that slowly changing conditions can cause quite abrupt changes in the functioning of a system (i.e., regime shifts; Scheffer and Carpenter 2003), and also in SESs such shifts have been observed (Walker and Meyers 2004). Such shifts do not necessarily cause equally abrupt changes in the state of the system, but can gradually alter the system state over long periods of time (Walker and Meyers 2004, Biggs et al. 2018). The resilience of a SES strongly depends on how it reacts to gradual or sudden changes (Walker et al. 2004), but also on the functioning of the system after it has undergone a transformation (i.e., transformability; Folke et al. 2010). Predicting how a SES will behave under changing conditions and what the system may look like after periods of change requires a thorough understanding of the system. However, due to numerous complex interactions and feedbacks in many SESs, such system understanding is usually difficult to obtain.

It has long been known that the direction and rate of change in many complex systems are not random, but determined by stable and unstable equilibria in the system (Holling 1973, Scheffer et al. 2001). We clarify this statement with a simple example of a wild fishery from which fish are harvested (Fig. 1). Only when the harvest rate is equal to the natural growth rate is the fish stock size constant over time; i.e., the system is in an equilibrium state. In all nonequilibrium states, the stock will increase or decrease, depending on whether there is a growth or a harvest surplus, respectively. Depending on the system's internal functions (e.g., growth and harvest functions; Hannesson 1983), the emerging equilibria can be either stable (e.g., Fig, 1A) or unstable (e.g., Fig. 1B), meaning that change in fish stock size is either toward or away from the equilibrium, respectively. When systems are driven by more complex functions, it is also possible that multiple stable equilibria arise (e.g., Fig. 1C), which is particularly the case in systems with positive feedbacks (Angeli et al. 2004). Stable equilibria have also been referred to as "attractors" (Scheffer et al. 2012, Bitterman and Bennett 2016). The state space in which system change is in the direction of a stable equilibrium is commonly termed a "basin of attraction" (Holling 1973) or a "valley" in a stability landscape (Scheffer et al. 2001). Unstable equilibria are also known as "hills" in stability landscapes (Scheffer et al. 2001), or "separatrix" when located between two stable equilibria (e.g., Fig. 1C; Dasgupta and Mäler 2003).

System internal functions are influenced by external system stressors (e.g., climate or economic change), which in turn can affect equilibria. To assess a system's resilience under changing conditions, it is thus important to identify the stable and unstable equilibria for multiple values of a system stressor. For instance, in systems with multiple stable equilibria, a small change in a system stressor can cause a regime shift, i.e., a transition from one basin of attraction into another (Scheffer et al. 2001, Biggs et al. 2018). Such transitions can strongly affect the functioning of SESs (Lade et al. 2013) and predicting the conditions under which they occur has become a widely studied topic (Scheffer et al. 2009, 2012, Polhill et al. 2016). The stressor thresholds at which regime

${ }^{1}$ Planning of Landscape and Urban Systems, Dept. of Civil, Environmental and Geomatic Engineering, ETH Zurich, Zurich, Switzerland, ${ }^{2}$ Flury \& Giuliani agricultural and regional economic consulting, Zurich, Switzerland, ${ }^{3}$ School of Human Evolution and Social Change and School of Sustainabilty, Arizona State University, Tempe, Arizona, USA 
Fig. 1. Example plots of annual harvest and growth rates for varying fish stock sizes in a wild fishery. In each plot, the harvest rates are defined by different production functions. For the plots (A) and (B), we assume that the growth rate is 5 fish for every 10 fish in the fish stock. In (A), the harvest rate increases exponentially with fish stock size. There is an equilibrium at a fish stock size of 600 (i.e., harvest rate $=$ growth rate). In this case, regardless of the fish stock size the direction of movement (black arrows) is always toward the equilibrium (i.e., stable equilibrium; green dot). In (B), we assume a constant harvest rate of 300 fish/year. Again, the fish stock size will remain constant if the size is exactly 600 , but in this case, any deviation from this equilibrium will result in a fish stock trend away from this equilibrium (i.e., unstable equilibrium; red dot). In (C), there is a linear production function and natural growth follows a sigmoidal function, which leads to a situation with two stable equilibria and one unstable equilibrium (green and red dots, respectively).

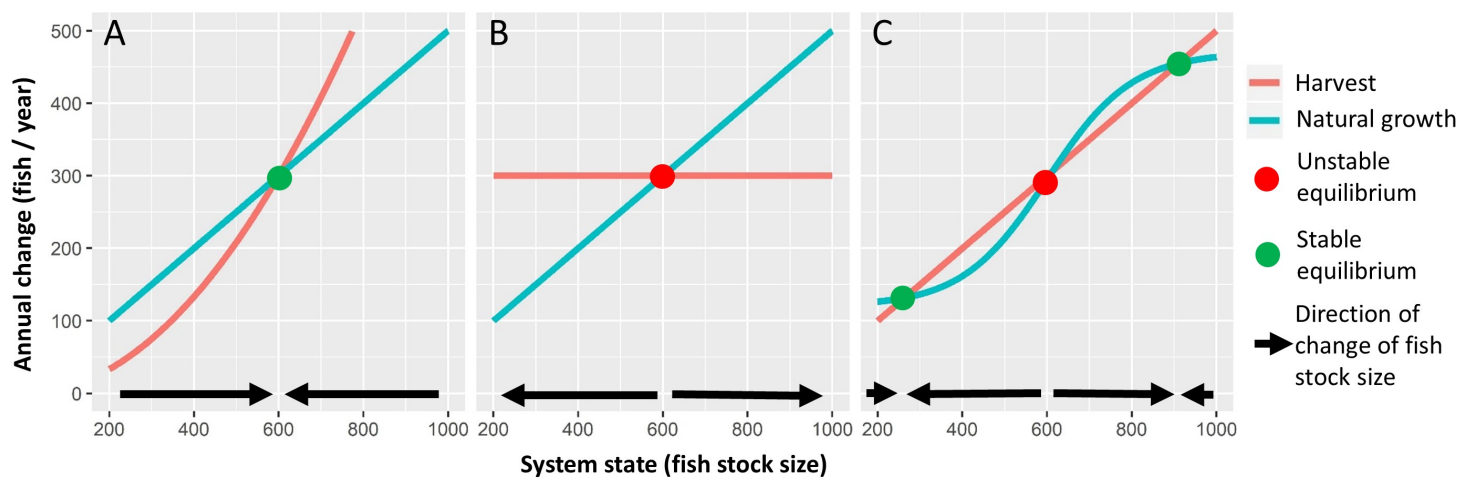

shifts take place can be derived by plotting bifurcation diagrams (i.e., plots of equilibria states against various values of a system stressor; e.g., Scheffer et al. 2001, Dasgupta and Mäler 2003). Another indicator of system resilience is how long it takes for a system to recover from sudden changes in stressors or in the system state (i.e., shocks; Folke 2006). This recovery time will also largely depend on the postshock state of the system in relation to its stable or unstable equilibrium (Veraart et al. 2012). System resilience may also be quantified by the magnitude of shocks that it can absorb (Walker et al. 2004). For such an analysis, the size of a basin of attraction has been proposed as an indicator (Holling 1973). Other resilience studies focus on the behavior of SES that are approaching equilibrium (i.e., transient dynamics; e.g., Fletcher and Hilbert 2007). For such studies, it is also important to know the equilibrium conditions of the system. Thus, from several perspectives, the assessment of the resilience of SESs depends on knowledge of equilibria and their behavior under changes in system stressors.

Despite their importance, equilibria in SESs are usually difficult to identify. If the internal system functions are well known, as in the fisheries example above, it may be possible to determine equilibria analytically. However, in many complex SESs these system functions are either unknown or too numerous for an analytical approach. Furthermore, gaining such system knowledge from real SESs is usually hampered by the lack of appropriate and sufficient data (but see Walker and Meyers 2004, Biggs et al. 2018). Models of SESs are capable of capturing the complexities found in real systems (Schlüter et al. 2012) and, in contrast to real systems, can easily be run with a range of input parameters. Agent-based SES models are ideally suited to study the complex system dynamics that emerges from the interactions between different actors and their environment (Schulze et al. 2017). However, studies in which agent-based SES models are presented, often focus on the model development, rather than on what can be learned from the models (Lee et al. 2015, Schulze et al. 2017). The analysis performed with agent-based SES models is generally rather limited: usually only few scenarios are tested (Filatova et al. 2016) and proper sensitivity analyses are rarely performed (Schulze et al. 2017). A notable exception is the study by Bitterman and Bennett (2016), who successfully identified stable equilibria with an agent-based land-use model. These authors repeatedly ran their model for many combinations of input parameters until, after approximately 20 time steps, an equilibrium was reached. However, depending on the type of external stressor, in many SESs transitions toward an equilibrium are much slower (Walker and Meyers 2004). For such systems, it would become too time consuming to perform many simulations over long time periods until an equilibrium state is reached.

In this study, we present an alternative approach to identify and quantify stable and unstable equilibria with agent-based SES models. Our approach does not require a model to reach an equilibrium state, but rather identifies equilibria from many shortterm runs. With our approach, we aim to add to the suite of methods that can be used to analyze output of agent-based models (Lee et al. 2015) and bridge the gap between SES modelling and resilience theory.

We demonstrate the approach using the agent-based land-use model ALUAM-AB, which simulates land-use changes in mountain landscapes based on land-use decisions of individual farmers under a range of socioeconomic, political, and ecological constraints (Brändle et al. 2015). With this model, we measured the direction of land-use change under different combinations of initial system states and levels of market, policy, and environmental stressors. The system state was expressed as the areas of intensive and extensive agriculture in the study region 
because these are important indicators for the ecological quality of an agricultural area. With direction-field plots and reconstructed stability landscapes (Peterson et al. 1998, Scheffer et al. 2001), we visually identified equilibria in both the areas of intensive and extensive agriculture. The equilibria were quantitatively analyzed with support-vector machine classifications (Shmilovici 2010) with which we plotted bifurcation diagrams. With these diagrams, we were able to capture the main behavior of our complex system in a simple metamodel. Finally, we discuss what the identified equilibria reveal about the functioning of the system and its resilience. The presented approach is generic and can be applied to other agent-based SES models. We emphasize that knowledge on equilibria can be used for regime-shift analysis, which is a central focus of SES modeling studies (Polhill et al. 2016), but also has a broader applicability in SES science (e.g., the existence of one stable equilibrium can help understand system dynamics).

\section{METHODS}

\section{Case study region}

The case study region $\left(443.3 \mathrm{~km}^{2}\right)$ is located in the central part of the Canton of Valais in Switzerland, which is a drought-sensitive, continental, inner-alpine mountain region. Unproductive ground, including rocks and glaciers, accounts for $62 \%$ of the area, while $20 \%$ is covered by forest, $16 \%$ by agriculture, and $2 \%$ by settlement. Small-scale farming practices, including seasonal alpine grazing, maintain a diverse and patchy landscape. On average, individual farmers cultivate only eight ha of agricultural land and keep around seven livestock units of which many are sheep. Agriculture is highly subsidized and federal reimbursements make up more than half of farmers' agricultural incomes. Over $90 \%$ of the farmers work part-time with additional jobs in tourism or industry (Brunner et al. 2016). Because the region is among the driest of the Swiss Alps, future changes in temperature and precipitation are predicted to increase vegetation and agricultural yields (Briner et al. 2012). These socioeconomic, political, and ecological boundary conditions make the region susceptible to changes in external stressors, such as prices for agricultural produce, subsidy policies, or climate (Grêt-Regamey et al. 2019). Therefore, we focussed on these three stressors in subsequent analyses.

\section{Agent-based alpine land use allocation model (ALUAM-AB)}

The land-use decisions taken by the farmers in our case study region were simulated with ALUAM-AB (Brändle et al. 2015). A description of ALUAM-AB following the overview, design concepts, and details (ODD) protocol (Grimm et al. 2006) is given in Appendix 1. In short, the model has been designed to simulate land-use change in mountain SESs considering the combined effects of climate, market, and policy changes and the behavior of farmers. Each agent in the model represents a group of farmers that has the same decision-making mechanisms for managing farm resources. The agents can practice both extensive and intensive farming depending on the conditions. The initial state of each agent is characterized by several parameters (e.g., land endowment and livestock capacity), which are updated after each yearly simulation period following the decisions of farmers. The farmers allocate their available resources to maximize their income, considering parcel characteristics (e.g., slope, elevation, or soil suitability), farm level, and individual constraints as well as external socioeconomic and political conditions. Once an optimal land-use allocation is reached, farm capacities, livestock, and age of the farmers are updated, and the next annual time step is initialized. Interaction between agents is simulated with an exchange of land units. The model identifies parcels that are no longer cultivated and either assigns them to other farmers, who can generate profit from the parcel and are willing to expand, or defines them as abandoned in which case they are subject to forest growth. Interaction between agents and the environment is simulated via a linkage with the "LandClim" model, which is a spatially explicit process-based model that simulates forest dynamics and yields on meadows given different management regimes and external conditions (Schumacher and Bugmann 2006).

In our study, ALUAM-AB was run with an initial regional community of 250 farmers (in 2001, there were 251 farmers in the study area), assigned to 1 of 14 agents/farmer types. The agent typologies were derived from interviews with 15 local farmers, a farm survey $(n=111)$ as well as an analysis of agricultural census data. Unproductive land was not considered in the modeling because the extreme topographic conditions make future management of these lands very unlikely. Each hectare of land was considered a parcel and the simulation included 12,163 of such parcels. The spatially explicit data for each parcel was obtained from a variety of sources (i.e., Swisstopo 2005, FOAG 2008, SFSO 2009).

\section{ALUAM-AB simulations}

For our analysis, we ran ALUAM-AB repeatedly for different combinations of initial system states and external stressors. To investigate effects of economic stressors, we varied market prices for agricultural commodities (e.g., prices for meat, milk, hay, and fodder). Changes in subsidy policies (i.e., policy stressor) were simulated by varying agricultural direct payments (e.g., payments for biodiversity, animal-friendly farming practices, and summer pastures). The influence of climate change (i.e., environmental stressor) was assessed by changing the yield of the parcels for different agricultural land uses. For each stressor, we created 350 input settings by making random combinations of initial system states and levels of the respective stressor, while keeping the other stressors fixed at their 2001 levels (i.e., baseline values calculated for ALUAM-AB). Stressor levels were varied by multiplying the baseline values of all variables belonging to a certain type of stressor with a randomly chosen multiplication factor ranging between 0.1 and 2.0. The area of extensive and intensive agriculture (i.e., our system states) are an emergent property of the model. Therefore, the initial land-use configuration was varied by randomly choosing shares of forest and summer pastures ranging between 5 and $95 \%$, while the remaining parcels were all assigned to agricultural land (without specifying whether it was intensive or extensive). Proportionate to these shares, the three land-uses were randomly assigned to parcels. We used a Montecarlo simulation with a uniform probability distribution to create our input settings because this produced a fairly continuous distribution of input settings throughout the state space. To make our results as generic as possible and not dependent on specific system settings, each farmer in each run was randomly assigned a farmer type, an age, and a number of parcels. Pre- and postprocessing of ALUAM-AB input and output was performed in R (R Development Core Team 2018). 
For each of the 1050 input settings, we ran ALUAM-AB over a 10-year period (2001-2010). To speed up this process, we created an R-script that automatically initialized a new run upon completion of the previous run and that was able to run ALUAM$\mathrm{AB}$ in parallel on multiple computer cores. Some of the parcels to which the land-uses forest, summer pasture, and agriculture were randomly assigned, were not suitable for the assigned landuse because of topographic constraints. Therefore, in the first simulation year, most model runs showed significant fluctuations in the land uses after which the land-use configuration stabilized. We therefore discarded the results from the first simulation year. In the second simulation year (i.e., 2002), we calculated the area of extensive and intensive agriculture, which determined our initial system states in subsequent analyses. With a linear regression analysis, we then determined the direction and rate of change in the area of extensive or intensive agriculture over a nineyear period (i.e., 2002-2010). The obtained regression coefficients indicated how much the area of intensive or extensive agriculture had increased or decreased per year. A nonsignificant coefficient $(p>0.05)$ was regarded as no change.

\section{Direction-field plots}

From the output of the ALUAM-AB runs, we created "directionfield plots" that depict changes in the area of intensive or extensive agriculture as a function of the value of a system stressor. The plots consist of arrows of which the coordinates of the starting point are the multiplication factor of the stressor (i.e., $x$-axis of the plot) and the area of extensive or intensive agriculture in 2002 (i.e., y-axis of the plot). The endpoint of the arrows along the yaxis represents the expected change in area of extensive or intensive agriculture over a 10 -year period obtained by multiplying the regression coefficients by 10 . Because the stressor levels were kept constant throughout a 10 -year simulation, the start- and endpoint along the $\mathrm{x}$-axis are equal (i.e., vertical line). These direction-field plots are similar to well-known vector-field plots (Boker and McArdle 2005), although in the latter the variables on both the $\mathrm{x}$ - and $\mathrm{y}$-axis can vary over time. In directionfield plots, the equilibria are located in those areas where there is a change in the predominant direction of the arrows. When arrows surrounding such areas are pointing away from the equilibrium, it is unstable. Alternatively, when arrows are pointing toward such areas, it is indicative of a stable equilibrium. We created separate direction-field plots for extensive and intensive agriculture as well as for each of the different stressor types. The R-code used to create the direction-field plots is supplied in Appendix 2.

\section{Reconstructing stability landscapes}

A stability landscape is a multidimensional state space in which stable equilibria are portrayed as valley bottoms and unstable equilibria as hill ridges. Over the past two decades, stability landscapes have frequently been used in resilience theory to exemplify the effects of changing equilibria and regime shifts (e. g., Peterson et al. 1998, Scheffer et al. 2001, Walker et al. 2004, Bitterman and Bennett 2016). In this study, we reconstructed the stability landscapes from the results of the ALUAM-AB runs. In a first step, we created a two-dimensional grid $(25 \times 25$ cells $)$ of the state space used for the direction-field plots. We then interpolated the regression coefficients with a moving window analysis (Gaussian weighting kernel), so that each grid cell contained a coefficient value. Subsequently, we calculated the cumulative sum of the interpolated regression coefficients in each column in the grid (i.e., along the y-axis) and rescaled the values per column between zero and one. These rescaled values represented the $\mathrm{z}$-axis in the stability landscape. We used the Rpackage plotly (Sievert et al. 2017) to create 3D interactive plots of the stability landscapes (R-code in Appendix 2).

Bifurcation diagrams with support-vector machine classifications In addition to the visual analysis of equilibria with direction-field plots and stability landscapes, we also performed a quantitative analysis with support-vector machines (SVMs). Over the past two decades, SVMs have become a popular statistical learning method for supervised classification (Shmilovici 2010). In the same state spaces as the direction-field plots, we used SVMs to define the separator line that best divides the data points into regions with positive and negative growth of intensive or extensive agriculture. This separator line is a quantitative representation of equilibrium states. To find this best separator line, we performed a crossvalidated grid-search of hyperparameters for the SVM classifier (gamma-values were varied between 0.01 and 1.0 and cost-values were varied between 0.01 and 2.0). As performance measure of the classifier, we used the classification error (i.e., proportion of falsely classified points). Because we were interested in regions of positive or negative growth, the few simulations without any growth were not considered in this analysis. We colored the separator line by hand to indicate stable and unstable equilibria. For the SVM analysis, we made use of the R-package e1071 (Meyer et al. 2017; R-code in Appendix 2).

\section{RESULTS}

Performing all 1050 runs with ALUAM-AB took approximately 2 weeks on 24 -core desktop computers. Because the directionfield plots showed comparable results for the three stressor types, we only show the results for the changes in agricultural direct payments (Fig. 2) and included the plots for the other stressor types in Appendix 3. The results table of model runs in which direct payments were varied is included in Appendix 4. For intensive agriculture, the direction-field plot shows an unstable equilibrium: with relatively small areas of intensive agriculture, the growth tends to be negative, while with relatively large areas, the predominant growth is positive (Fig. 2A). This result implies that there are probably two stable equilibria for the area of intensive agriculture on either side of the unstable equilibrium, but the location of these equilibria is not so apparent from the direction-field plot (Fig. 2A). An opposite pattern can be seen for extensive agriculture, in which there is a negative growth with relatively large areas of extensive agriculture and a positive growth with small areas of extensive agriculture (Fig. 2B). Thus, for extensive agriculture a stable equilibrium emerges. For both intensive and extensive agriculture, the unstable and stable equilibrium state increases with increasing direct payments (Fig. 2) as well as with increasing market prices (Appendix 3, Fig. A3.1). Only with increasing yields (i.e., environmental stressor), did we find that the equilibrium states for both intensive and extensive agriculture decreased (Appendix 3, Fig. A3.1). The same patterns of equilibria can also be seen in the top-down views of the reconstructed stability landscapes (Fig. 3A, B). The sideperspectives of the interactive stability landscapes (Fig. 3C, D) allow for an easier identification of the hills and valleys. 
Fig. 2. Direction-field plots of the direction and magnitude of change in the area of intensive or extensive agriculture under different levels of direct payments. Each arrow originates from the point that represents the initial area of intensive (A) or extensive (B) agriculture and the multiplication factor with which the direct payments were multiplied (baseline $=1$ ). The arrow represents the 10-year trend of change in agricultural area. The arrows can indicate a positive (green), negative (red), or no (white) trend. The intensity of the red and green colors increases with an increasing magnitude of change.

\section{A}

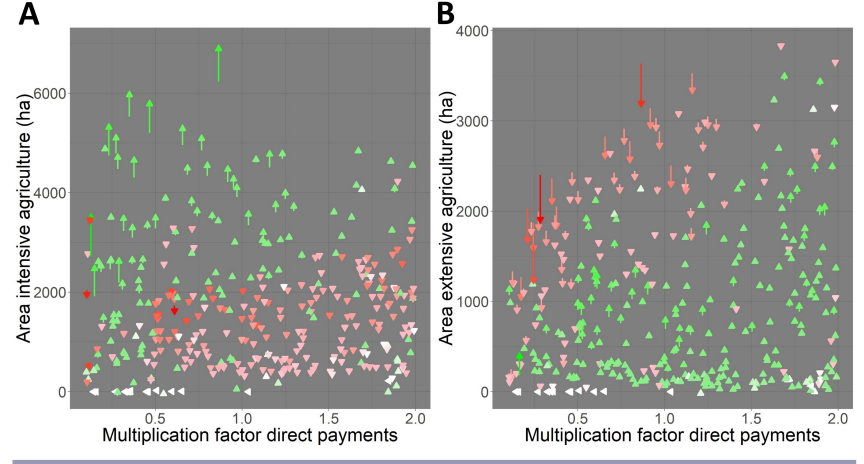

The SVM classification (Fig. 4) had an average classification error of $18.1 \%$ for all stressor and agriculture types (Table 1). From the bifurcation diagrams, we observed that the relationship between the equilibrium states and the level of system stressor is not linear (Fig. 4). The general trends from the direction-field plots can also be observed in the bifurcation diagrams. However, the latter suggests that both intensive and extensive agriculture contain "limit points," which are points on the separator line where the tangent is vertical and the equilibria switches from stable to unstable, or vice versa (e.g., Qi et al. 2015; Fig. 4). For instance, with intensive agriculture and a multiplication factor for direct payments of 0.5 , there is a stable and an unstable equilibrium (Fig. 4A). Furthermore, for relatively low levels of direct payments, irrespective of the initial area of intensive or extensive agriculture, the growth direction is predominantly positive or negative, respectively (Fig. 4). Only when the direct payments surpass a certain threshold (i.e., multiplication factor $>0.4$ for intensive agriculture and $>0.2$ for extensive agriculture), do the equilibria emerge (Fig. 4). Such a threshold was not observed for market prices nor for yields (Appendix 3, Fig. A3.2). The shapes of the separator lines in the bifurcation diagrams of intensive (Fig. 3A) and extensive agriculture (Fig. 3B) correspond to the hill ridge (Fig. 4A) and valley bottom (Fig. 4B), respectively, in the stability landscapes.

\section{DISCUSSION}

In this study, we demonstrated how stable and unstable equilibria can be identified in the output of agent-based models of SESs with direction-field plots and bifurcation diagrams. The produced stability landscapes are handy visual aids to convey the results of our analyses. Knowledge on equilibria states and on their reaction to changes in external system stressors are vital to understand the resilience of SESs. For example, from our results we can learn that an increase in direct payments can cause the area of intensive
Fig. 3. Three-dimensional stability landscapes reconstructed from development trends of the area of intensive $(\mathrm{A}, \mathrm{C})$ or extensive (B, D) agriculture under different levels of direct payments. The $\mathrm{x}$ - and $\mathrm{y}$-axes are the same as in Figure 2. The $\mathrm{z}$ axis is unitless and ranges between zero and one. To reconstruct the landscapes, the detected trends (Fig. 2) were interpolated with a moving window analysis over the complete state space. In (A) and (B), there is a top-down perspective on the landscapes and it can be seen that the hill ridge (A) or valley bottom (B) correspond to the identified equilibria (Fig. 4). In (C) and (D), a side-perspective of the landscapes is shown and the hill (C; i.e., unstable equilibrium) and valley (D; i.e., stable equilibrium) can clearly be identified. These perspectives are screenshots from 3D interactive plots produced with Plotly (Plotly Technologies Inc., Montréal, Canada).

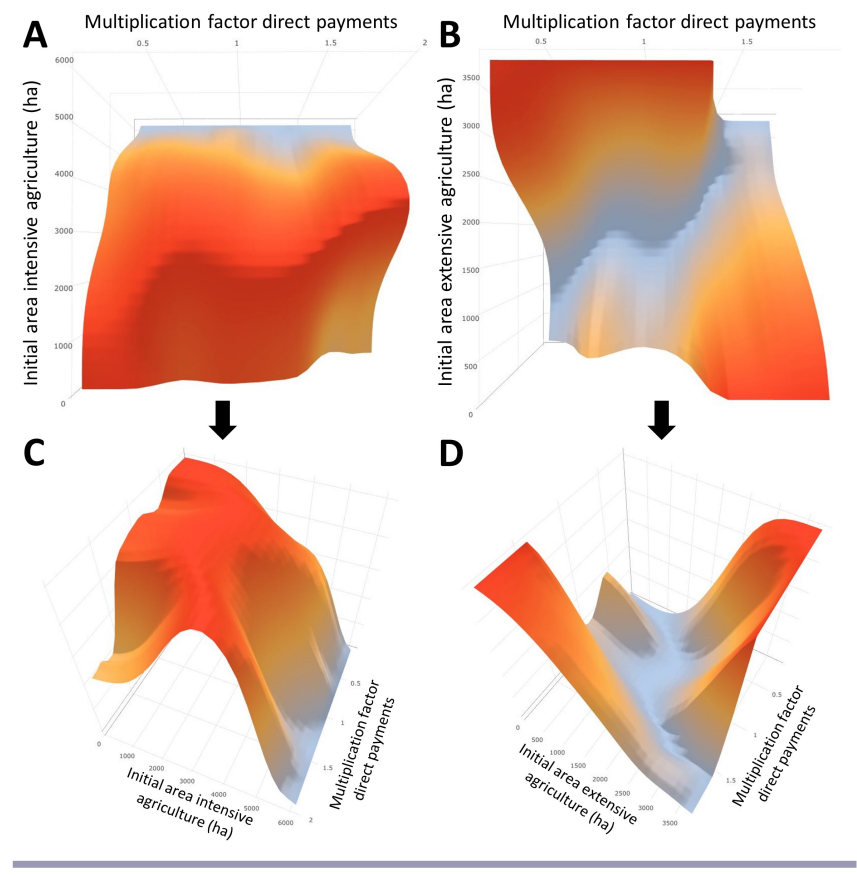

agriculture to switch from a situation of growth to one of shrinkage (i.e., regime shift). The separator line in the bifurcation diagram can be used to estimate the value of direct payments that will cause a system with a certain area of intensive agriculture to exhibit such a regime shift (Fig. 3A). The detected limit points can also help understand complex system behavior. For example, if the system state of intensive agriculture is below the limit point ( $<$ approx. 1300 ha; Fig. 4A) and the multiplication factor of direct payments is above it ( $>$ approx. 0.4 ; Fig $4 \mathrm{~A}$ ), increasing the direct payments will always lead to a reduction of the intensive agriculture. Only if one increases the area of intensive agriculture to above the unstable equilibrium, will an increase in direct payments lead to a growth in intensive agriculture. We also discovered that a minimum level of direct payments is necessary to maintain or increase extensive agriculture in our study area. With low levels of direct payments, the area of extensive agriculture is always decreasing whereas the area of intensive agriculture is increasing. 
Table 1. Results of the support-vector machine (SVM) classification of areas of positive and negative growth in extensive or intensive agriculture. The various external system stressors were varied together with the initial landscape composition to create a state space. For each model run, we assessed the direction of growth for the area of intensive and extensive agriculture. The classification error indicates the proportions of wrongly classified model runs (i.e., falsely classified data points) in the best performing model. The latter was determined with a 10 -fold crossvalidation grid search of the hyperparameters cost and gamma (those belonging to the best model are listed).

\begin{tabular}{lcccc}
\hline \hline $\begin{array}{l}\text { External system } \\
\text { stressor }\end{array}$ & $\begin{array}{c}\text { Agriculture } \\
\text { type }\end{array}$ & $\begin{array}{c}\text { Classification } \\
\text { error }\end{array}$ & Cost & Gamma \\
\hline Direct payments & Extensive & 0.182 & 2.000 & 1.000 \\
Direct payments & Intensive & 0.182 & 0.863 & 1.000 \\
Price & Extensive & 0.162 & 1.431 & 0.151 \\
Price & Intensive & 0.227 & 1.147 & 0.010 \\
Yield & Extensive & 0.147 & 2.000 & 0.151 \\
Yield & Intensive & 0.186 & 0.579 & 0.293 \\
\hline
\end{tabular}

Fig. 4. Bifurcation diagrams created from support-vector machine (SVM) classifications of areas of positive and negative growth of intensive (A) or extensive (B) agriculture. The points in the plots are the same as the starting points of the arrows in Figure 2. The growth trend in each model run was assigned to either of three classes: positive (green dot), negative (red dot), or flat (grey dot). The positive and negative trends were used to train SVMs to find the equilibria (i.e., separator line) between areas of positive (green area) and negative (red areas) growth. We colored the separator line by hand to indicate stable (blue line) or unstable (orange line) equilibria.

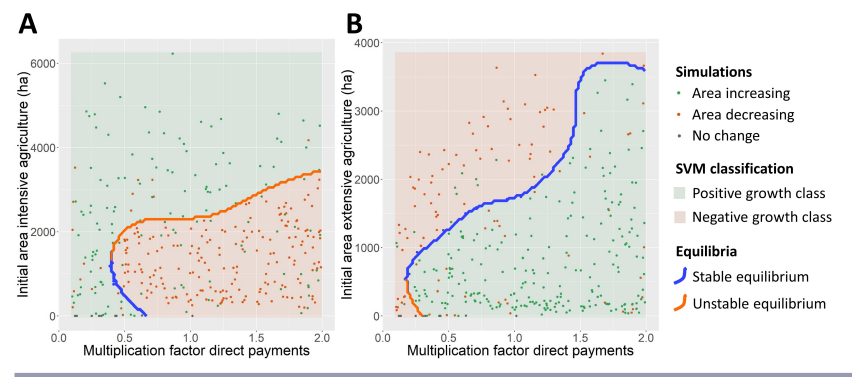

By comparing our results to expected outcomes, our results can uncover unexpected system behavior, but also serve as a validation of the agent-based model. For example, in Swiss mountain areas, agriculture in general is dependent on direct payments, and this is especially the case for extensive agriculture (Flury et al. 2005). Therefore, it is not surprising that the area of extensive agriculture is likely to decrease with very low direct payments. The fact that we found a clear unstable equilibrium for intensive agriculture and a stable equilibrium for extensive agriculture, suggests that these two agricultural practices are in trade-off (i.e., an increase of extensive agriculture goes together with a decrease in intensive agriculture, and vice versa). Although the existence of such a trade-off makes sense given the limited area of arable land in the study region, a situation in which all arable land is abandoned and overgrown with forest may have also been plausible given the dependency of both intensive and extensive agriculture on direct payments. The latter apparently does not happen even at low levels of direct payments. However, we have not experimented with direct payments below $10 \%$ of current levels.

The approach we presented is generic and in principle can be applied to any agent-based model. One advantage of our approach is that it does not require models to be run until equilibrium states have been reached. This makes the approach applicable to large models with long simulation times, which is a characteristic of many agent-based SES models. In our study, we chose to run ALUAM-AB for 10 time-steps (i.e., 10-year periods), so that we could perform enough simulations within an acceptable time. An alternative approach would be to run fewer simulations for longer time periods until stable equilibria have been reached, as was, for instance, done by Bitterman and Bennett (2016). However, in some models it can take many time-steps until equilibria states are reached and a single simulation would become too time consuming. Nonetheless, with our approach it remains unclear whether the equilibria that are identified from simulations over a limited number of time-steps are also the equilibria that would finally be achieved after simulating many time-steps. In fact, the comparison of short-term equilibria (e.g., with the approach presented here) and long-term equilibria (e.g., with the approach from Bitterman and Bennett 2016) can provide valuable information of the temporal stability of equilibrium states. As we show in our results, external system stressors can considerably affect the equilibrium states in our SES. However, system-internal interactions and feedbacks could also cause changes to the equilibrium states over time. The existence of such temporal changes in equilibrium states can be assessed by making bifurcation diagrams for different time lags during the simulation.

Stochasticity in the output of agent-based models often complicates the analysis and interpretation of the results (Lee et al. 2015). Despite the fact that every model run was initialized with randomized input settings, equilibria were still clearly identifiable from the direction-field plots and bifurcation diagrams. This suggests that the results from ALUAM-AB are not very stochastic, which has also been observed in other studies using this model (Brändle et al. 2015). In highly stochastic systems, there will not be a single direction of change, but a range of outcomes. Nevertheless, "even if stochasticity is large, systems will more often be found close to attractors than far away from them" (Scheffer et al. 2012:346). To detect such attractors in highly stochastic agent-based SES models, it is necessary to perform repeated model runs for each set of input settings. For each input setting, the statistical moments of the change in system state (i. e., mean, variance, and skewedness of the change) can then be calculated. In a direction-field plot, instead of plotting a single arrow for each model run, one could plot multiple arrows from which the predominant direction of change can also be inferred. In models of highly stochastic systems with multiple basins of attraction, repeated model runs can be drawn toward different attractors; a phenomenon known as "flickering" (Scheffer et al. 2009). If such flickering is observed it can be an indication of an unstable equilibrium state. 
In the SVM classification of model runs leading to positive or negative growth (Fig. 4), we found that the classification was not perfect and that on average $18 \%$ of the data points were wrongly classified. On the one hand, this classification error could be due to stochasiticity in our model results, which we expect is not so high (see above). On the other hand, the wrongly classified points could be due to certain (emergent) system properties that cause the atypical growth trends. In the latter case, discovering these system properties could provide information on how the growth trend in SESs can be changed to steer the system in a desired direction.

Recently, several authors have expressed their concerns about the lack of guidelines for the analysis and description of output of agent-based SES models (Angus and Hassani-Mahmooei 2015, Lee et al. 2015, Filatova et al. 2016, O'Sullivan et al. 2016, Schulze et al. 2017). The lack of such guidelines hinders the transferability of knowledge and results between models (Schulze et al. 2017) and can lead to the situation in which each agent-based model is so case-specific that it becomes hard to compare models and draw general inferences from output of several models (O'Sullivan et al. 2016). The complexity of many agent-based models and the large amount of model output makes it difficult to distil and present the most important results in an understandable way (Lee et al. 2015). The approach presented here allows the summarizing of important model results (i.e., equilibria states and their reactions to changing conditions) in a condensed and understandable format. Given that the approach is generic, it can be applied to the output to multiple agent-based and nonagentbased SES models, which will facilitate the transferability of model results. An important tool to evaluate agent-based models are sensitivity analyses, which involve assessing the effect of model output on changes in input parameters (O'Sullivan et al. 2016, Schulze et al. 2017). With few minor adaptations, our approach can thus be easily incorporated into traditional sensitivity analyses. Finally, because unstable and stable equilibria play an essential role in the resilience of SESs, the presented plots (i.e., direction-field plots, bifurcation diagrams, and stability landscapes) can be used to convey information about system resilience and bridge the gap between SES models and resilience theory. We anticipate that a stronger focus on the identification of equilibria in agent-based SES models and their comparison between models can avoid what O'Sullivan et al. (2016) referred to as the YAAWN syndrome ("Yet another agent-based model... whatever...nevermind...").

Responses to this article can be read online at: http://www.ecologyandsociety.org/issues/responses. $\mathrm{php} / 10899$

\footnotetext{
Acknowledgments:

We thank Robert Huber for his valuable advice about ALUAM-AB. We also thank Julia Klein and the Mountain Sentinels Collaborative Network for bringing the authors together in a workshop on participatory modelling. This study was part of the MntPaths project ("Pathways for global change adaptation of mountain socioecological systems") financed by the Swiss National Science Foundation (Grant nr. 20521L_169916).
}

\section{LITERATURE CITED}

Angeli, D., J. E. Ferrell, Jr. and E. D. Sontag. 2004. Detection of multistability, bifurcations, and hysteresis in a large class of biological positive-feedback systems. Proceedings of the National Academy of Sciences 101:1822-1827. https://doi.org/10.1073/ pnas. 0308265100

Angus, S. D., and B. Hassani-Mahmooei. 2015. "Anarchy” reigns: a quantitative analysis of agent-based modelling publication practices in JASSS, 2001-2012. Journal of Artificial Societies and Social Simulation 18(4):16. https://doi.org/10.18564/jasss.2952

Biggs, R., G. D. Peterson, and J. C. Rocha. 2018. The regime shifts database: a framework for analyzing regime shifts in socialecological systems. Ecology and Society 23(3):9. https://doi. org/10.5751/ES-10264-230309

Bitterman, P., and D. A. Bennett. 2016. Constructing stability landscapes to identify alternative states in coupled socialecological agent-based models. Ecology and Society 21(3):21. https://doi.org/10.5751/ES-08677-210321

Boker, S. M., and J. J. McArdle. 2005. Vector field plot. Pages 5700-5704 in P. Armitage and T. Colton, editors. Encyclopedia of biostatistics. Wiley, Hoboken, New Jersey, USA. https://doi. org/10.1002/0470011815.b2a12068

Brändle, J. M., G. Langendijk, S. Peter, S. H. Brunner, and R. Huber. 2015. Sensitivity analysis of a land-use change model with and without agents to assess land abandonment and long-term re-forestation in a Swiss mountain region. Land 4:475-512. https:// doi.org/10.3390/land4020475

Briner, S., C. Elkin, R. Huber, and A. Grêt-Regamey. 2012. Assessing the impacts of economic and climate changes on landuse in mountain regions: a spatial dynamic modeling approach. Agriculture, Ecosystems, and Environment 149:50-63. https://doi. org/10.1016/j.agee.2011.12.011

Brunner, S. H., R. Huber, and A. Grêt-Regamey. 2016. A backcasting approach for matching regional ecosystem services supply and demand. Environmental Modelling and Software 75:439-458. https://doi.org/10.1016/j.envsoft.2015.10.018

Dasgupta, P., and K.-G. Mäler. 2003. The economics of nonconvex ecosystems: introduction. Environmental and Resource Economics 26:499-525. https://doi.org/10.1023/B:

EARE.0000007347.37345.55

Federal Office for Agriculture (FOAG). 2008. Swiss agricultural census data 2000-2012. Federal Office for Agriculture, Bern, Switzerland.

Filatova, T., J. G. Polhill, and S. van Ewijk. 2016. Regime shifts in coupled socio-environmental systems: review of modelling challenges and approaches. Environmental Modelling and Software 75:333-347. https://doi.org/10.1016/j.envsoft.2015.04.003

Fletcher, C. S., and D. W. Hilbert. 2007. Resilience in landscape exploitation systems. Ecological Modelling 201:440-452. https:// doi.org/10.1016/j.ecolmodel.2006.10.011

Flury, C., N. Gotsch, and P. Rieder. 2005. Site-specific and regionally optimal direct payments for mountain agriculture. Land Use Policy 22:207-214. https://doi.org/10.1016/j.

landusepol.2004.02.003 
Folke, C. 2006. Resilience: the emergence of a perspective for social-ecological systems analyses. Global Environmental Change 16:253-267. https://doi.org/10.1016/j.gloenvcha.2006.04.002

Folke, C., S. R. Carpenter, B. Walker, M. Scheffer, T. Chapin, and J. Rockström. 2010. Resilience thinking: integrating resilience, adaptability and transformability. Ecology and Society 15(4):20. https://doi.org/10.5751/ES-03610-150420

Grêt-Regamey, A., S. H. Huber, and R. Huber. 2019. Actors' diversity and the resilience of social-ecological systems to global change. Nature Sustainability 10.1038/s41893-019-0236-z. https:// doi.org/10.1038/s41893-019-0236-Z

Grimm, V., U. Berger, F. Bastiansen, S. Eliassen, V. Ginot, J. Giske, J. Goss-Custard, T. Grand, S. K. Heinz, G. Huse, A. Huth, J. U. Jepsen, C. Jørgensen, W. M. Mooij, B. Müller, G. Pe'er, C. Piou, S. F. Railsback, A. M. Robbins, M. M. Robbins, E. Rossmanith, N. Rüger, E. Strand, S. Souissi, R. A. Stillman, R. Vabø, U. Visser, and D. L. DeAngelis. 2006. A standard protocol for describing individual-based and agent-based models. Ecological Modelling 198:115-126. https://doi.org/10.1016/j.ecolmodel.2006.04.023

Hannesson, R. 1983. Bioeconomic production function in fisheries: theoretical and empirical analysis. Canadian Journal of Fisheries and Aquatic Sciences 40:968-982. https://doi. org/10.1139/f83-123

Holling, C. S. 1973. Resilience and stability of ecological systems. Annual Review of Ecology and Systematics 4:1-23. https://doi. org/10.1146/annurev.es.04.110173.000245

Lade, S. J., A. Tavoni, S. A. Levin, and M. Schlüter. 2013. Regime shifts in a social-ecological system. Theoretical Ecology 6:359-372. https://doi.org/10.1007/s12080-013-0187-3

Lee, J.-S., T. Filatova, A. Ligmann-Zielinska, B. HassaniMahmooei, F. Stonedahl, I. Lorscheid, A. Voinov, G. Polhill, Z. Sun, and D. C. Parker. 2015. The complexities of agent-based modeling output analysis. Journal of Artificial Societies and Social Simulation 18(4):4. https://doi.org/10.18564/jasss.2897

Meyer, D., E. Dimitriadou, K. Hornik, A. Weingessel, F. Leisch, C.-C. Chang, and C. C. Lin. 2017. e1071: misc functions of the Department of Statistics, Probability Theory Group (Formerly: E1071), TU Wien. R-package version 1.6-8. R Foundation for Statistical Computing, Vienna, Austria. [online] URL: https:// rdrr.io/rforge/e1071/

O’Sullivan, D., T. Evans, S. Manson, S. Metcalf, A. LigmannZielinska, and C. Bone. 2016. Strategic directions for agent-based modeling: avoiding the YAAWN syndrome. Journal of Land Use Science 11:177-187. https://doi.org/10.1080/1747423X.2015.1030463

Peterson, G., C. R. Allen, and C. S. Holling. 1998. Ecological resilience, biodiversity, and scale. Ecosystems 1:6-18. https://doi. org/10.1007/s100219900002

Polhill, J. G., T. Filatova, M. Schlüter, and A. Voinov. 2016. Modelling systemic change in coupled socio-environmental systems. Environmental Modelling and Software 75:318-332. https://doi.org/10.1016/j.envsoft.2015.10.017

Qi, Z., X. Kong, and G. Wang. 2015. Identifying critical loads of frame structures with equilibrium equations in rate form.
Mathematical Problems in Engineering 2015:325969. https://doi. org/10.1155/2015/325969

R Development Core Team. 2018. R: a language and environment for statistical computing. R Foundation for Statistical Computing, Vienna, Austria. [online] URL: https://www.r-project.org/

Scheffer, M., J. Bascompte, W. A. Brock, V. Brovkin, S. R. Carpenter, V. Dakos, H. Held, E. H. van Nes, M. Rietkerk, and G. Sugihara. 2009. Early-warning signals for critical transitions. Nature 461:53-59. https://doi.org/10.1038/nature08227

Scheffer, M., and S. R. Carpenter. 2003. Catastrophic regime shifts in ecosystems: linking theory to observation. Trends in Ecology and Evolution 18:648-656. https://doi.org/10.1016/j. tree.2003.09.002

Scheffer, M., S. Carpenter, J. A. Foley, C. Folke, and B. Walker. 2001. Catastrophic shifts in ecosystems. Nature 413:591-596. https://doi.org/10.1038/35098000

Scheffer, M., S. R. Carpenter, T. M. Lenton, J. Bascompte, W. Brock, V. Dakos, J. van de Koppel, I. A. van de Leemput, S. A. Levin, E. H. van Nes, M. Pascual, and J. Vandermeer. 2012. Anticipating critical transitions. Science 338:344-348. https://doi. org/10.1126/science. 1225244

Schlüter, M., R. R. J. McAllister, R. Arlinghaus, N. Bunnefeld, K. Eisenack, F. Hölker, E. J. Milner-Gulland, B. Müller, E. Nicholson, M. Quaas, and M. Stöven. 2012. New horizons for managing the environment: a review of coupled social-ecological systems modeling. Natural Resource Modeling 25:219-272. https://doi.org/10.1111/j.1939-7445.2011.00108.x

Schulze, J., B. Müller, J. Groeneveld, and V. Grimm. 2017. Agentbased modelling of social-ecological systems: achievements, challenges, and a way forward. Journal of Artificial Societies and Social Simulation 20(2):8. https://doi.org/10.18564/jasss.3423

Schumacher, S., and H. Bugmann. 2006. The relative importance of climatic effects, wildfires and management for future forest landscape dynamics in the Swiss Alps. Global Change Biology 12:1435-1450. https://doi.org/10.1111/j.1365-2486.2006.01188.x

Shmilovici, A. 2010. Support vector machines. Pages 231-247 in O. Maimon, and L. Rokach, editors. Data mining and knowledge discovery handbook. Springer, Boston, USA.

Sievert, C., C. Parmer, T. Hocking, S. Chamberlain, K. Ram, M. Corvellec, and P. Despouy. 2017. plotly: create interactive web graphics via 'plotly.js'. R package version 4.7.1. R Foundation for Statistical Computing, Vienna, Austria. [online] URL: https:// rdrr.io/cran/plotly/

Swiss Federal Office of Topography (Swisstopo). 2005. Digital elevation model DHM 25. Swiss Federal Office of Topography, Wabern, Switzerland.

Swiss Federal Statistics Office (SFSO). 2009. Arealstatistik der Schweiz. Swiss Federal Statistics Office, Bern, Switzerland.

Veraart, A. J., E. J. Faassen, V. Dakos, E. H. van Nes, M. Lürling, and M. Scheffer. 2012. Recovery rates reflect distance to a tipping point in a living system. Nature 481:357-359. https://doi. org/10.1038/nature 10723 
Walker, B., C. S. Holling, S. R. Carpenter, and A. Kinzig. 2004. Resilience, adaptability and transformability in social-ecological systems. Ecology and Society 9(2):5. https://doi.org/10.5751/ ES-00650-090205

Walker, B., and J. A. Meyers. 2004. Thresholds in ecological and social-ecological systems: a developing database. Ecology and Society 9(2):3. https://doi.org/10.5751/ES-00664-090203 
Purpose. The purpose of the Agent-Based Alpine Land Use Allocation Model (ALUAM-AB) is to simulate the effect of socio-economic, climatic and political pressures on farm structure and emerging land-use changes in mountain landscapes.

State variables. Agents represent groups of farmers. An agent has (1) its own state (i.e., land endowment, stable capacity, etc.) which is updated after each yearly simulation period and (2) its own decision-making mechanisms for managing farm resources in form of constraints to an income optimization approach. Agent typologies were derived from interviews with and a survey among local farmers and from an analysis of agricultural census data. Fourteen agent types were derived from the farm survey using a PCA with a quartimax rotation and subsequent k-means clustering on 19 farmers' characteristics, which included the opportunity costs of labor, additional workforce hired, a threshold for minimum income, farm size, the intention to increase farm size or livestock housing capacity (details are given in Brändle et al. 2015). The median characteristics for each agent were then fed into the model (Table A1).

Scale. The smallest landscape unit is one hectare. The size of the study is 44330 ha, of which 12163 ha were used in the simulations. The model was run between 2001 and 2010.

Process overview and scheduling. ALUAM-AB proceeds in annual time steps. The agents allocate their available resources to maximize their income. Thereby they consider spatiallyexplicit natural, farm level and individual constraints as well as incentives and regulations from the market and policy instruments, which are annual input data to the model. Investments in production capacity made in previous years are considered as sunk costs representing path dependencies of the individual agents. Structural change is modeled using a land market module (Lauber 2006; Huber et al. 2013). The module identifies land units that are no longer cultivated under the existing farm structure due to negative land rents, because an agent does not reach the minimum wage level or if farmers retire without successor. The land market module randomly assigns the land units to one of the other agents and then checks whether the shadow price for the land unit is positive. This procedure is repeated until all land units are assigned to an agent or until no more agents are willing to take the land units left on the market. In that latter case, they are regarded as abandoned land and subject to natural vegetation dynamics. When land-use allocation is optimal, farm capacities and livestock, as well as the age of the agents are updated and the next annual time step is initialized.

Emergence. Changes in the activities of agents emerge from changes in prices, policies and the climate (see below scenarios) and depend on the decision-making type. In addition, land-use patterns emerge from structural changes at the agent level and from spatially explicit climateinduced changes of yield quantities.

Adaptation. Agents respond to external pressures by adjusting their production activities, applying new production technologies (e.g. irrigation), increasing (or reducing) land size and adjusting land-use intensities. In addition, agents exit the sector if their income falls below a minimum threshold.

Prediction. The model follows an income optimization approach assuming rational economic behavior with no direct learning pattern. However, the consideration of individual constraints, such as opportunity costs, minimum income wage and limited time resources, includes noneconomic goals in the decision-making process. 
Interaction. The interaction between agents is based on the land market described above. Interaction between agents and the environment is based on the model linkage with a submodel LandClim, which is a spatially-explicit process-based model that simulates forest dynamics and yields on meadows given different management regimes (Schumacher and Bugmann 2006).

Initialization. Initial attributes for agents were chosen randomly. This includes the age structure of each agent, the number of farmers in each agent and the allocation of land units to agents.

Input. Spatially-explicit data were derived from national data sets (Swisstopo 2005; FOAG 2008; SFSO 2009) or simulated with LandClim. In the baseline setting, policy and socioeconomic parameters were chosen to represent the local conditions in 2001 (Briner et al. 2012; Huber et al. 2014).

Calibration and validation. ALUAM-AB was validated against observed livestock and landuse data between 2001 and 2013. Overall and unequal variation errors of model performance were small (on average 6.5\%), thus, the model satisfactorily captured the mean and trends of the observed data (Brändle et al. 2015).

Software requirements. ALUAM-AB runs on Linear Programing Language (LPL) from Virtual Optima and requires ILOG CPLEX Optimization Studio from IBM. LPL academic license is available for purchase at http://www.virtual-optima.com/en/index.html, and CPLEX academic license is available free of change at https://www.ibm.com/software/. 
Table A1. Agent types in ALUAM-AB. Farm sizes, age of the farmers and number of farms in each agent were randomized.

\begin{tabular}{|c|c|c|c|c|c|c|c|c|c|}
\hline Agent & $\begin{array}{l}\text { Opportunity } \\
\text { costs }\end{array}$ & $\begin{array}{l}\text { Available } \\
\text { work }\end{array}$ & $\begin{array}{l}\text { Min. } \\
\text { income }\end{array}$ & $\begin{array}{l}\text { Farm } \\
\text { growth }\end{array}$ & Succession rate & Sheep & $\begin{array}{l}\text { Dairy } \\
\text { cows }\end{array}$ & $\begin{array}{l}\text { Beef } \\
\text { cattle }\end{array}$ & $\begin{array}{l}\text { Suckler } \\
\text { cows }\end{array}$ \\
\hline & x $10 \mathrm{CHF} / \mathrm{h}$ & $\%$ of $2800 \mathrm{~h}$ & $\mathrm{CHF}$ & & in $\%$ & \multicolumn{4}{|c|}{ Number in the year 2000} \\
\hline 1 & 0.2 & 1 & 25000 & Yes & 0.75 & & 237 & 215 & \\
\hline 2 & 0.2 & 0.6 & 25000 & Yes & 0.75 & 376 & & & \\
\hline 3 & 0.5 & 0.6 & 25000 & Yes & 0.75 & & & 86 & 43 \\
\hline 4 & 0.2 & 0.5 & 10000 & No & 0.55 & & 156 & 123 & \\
\hline 5 & 0.2 & 0.5 & 10000 & No & 0.55 & & 93 & 93 & \\
\hline 6 & 0.2 & 0.8 & 10000 & No & 0.45 & 44 & 41 & 208 & \\
\hline 7 & 0.2 & 0.5 & 100000 & No & 0.45 & 870 & & & \\
\hline 8 & 0.2 & 0.8 & 10000 & No & 0.45 & 26 & 27 & 146 & \\
\hline 9 & 0.5 & 0.5 & 0 & No & 0.45 & 208 & & & \\
\hline 10 & 0.5 & 0.5 & 0 & No & 0.45 & 222 & & & \\
\hline 11 & 1.25 & 0.3 & 0 & Yes & 0.55 & 558 & & & \\
\hline 12 & 1 & 0.5 & 10000 & Yes & 0.55 & & 38 & 27 & \\
\hline 13 & 1 & 0.3 & 10000 & No & 0.55 & & & & \\
\hline 14 & 0.2 & 0.3 & 0 & No & 0.45 & 932 & & & \\
\hline
\end{tabular}

\section{Literature cited}

Brändle, J., S. Peter, G. Langendijk, S. H. Brunner, and R. Huber, 2015. Sensitivity analysis of a land-use change model with and without agents to assess land abandonment and long-term re-forestation in a Swiss mountain region. Land 4:475-512.

Briner, S., C. Elkin, and R. Huber, 2012. Assessing the impacts of economic and climate changes on land-use in mountain regions: A spatial dynamic modeling approach. Agric Ecosyst Environ 149:50-63.

FOAG, 2008. Swiss Agricultural Census Data 2000-2012. Federal Office for Agriculture, Bern, Switzerland.

Grimm, V., U. Berger, F. Bastiansen, S. Eliassen, V. Ginot, J. Giske, J. Goss-Custard, T. Grand, S. K. Heinz, G. Huse, A. Huth, J. U. Jepsen, C. Jørgensen, W. M. Mooij, B. Müller, G. Pe'er, C. Piou, S. F. Railsback, A. M. Robbins, M. M. Robbins, E. Rossmanith, N. Rüger, E. Strand, S. Souissi, R. A. Stillman, R. Vabø, U. Visser, and D. L. DeAngelis, 2006. A standard protocol for describing individual-based and agent-based models. Ecological Modelling 198:115-126.

Huber, R., S. Briner, H. Bugmann, C. Elkin, C. Hirschi, R. Seidl, R. Snell, and A. Rigling, 2014. Inter- and transdisciplinary perspective on the integration of ecological processes into ecosystem services analysis in a mountain region. Ecological Processes 3:9.

Huber, R., S. Briner, A. Peringer, S. Lauber, R. Seidl, A. Widmer, F. Gillet, A. Buttler, Q. B. Le, and C. Hirschi, 2013. Modeling social-ecological feedback effects in the implementation of payments for environmental services in pasture-woodlands. Ecology and Society 18(2): 41 
Lauber, S., 2006. Agrarstrukturwandel im Berggebiet. ART Schriftenreihe. Dissertation ETH Nr.16716, Zürich, pp. 1 - 217.

Schumacher, S., and H. Bugmann, 2006. The relative importance of climatic effects, wildfires and management for future forest landscape dynamics in the Swiss Alps. Global Change Biology 12:1435-1450.

SFSO, 2009. Arealstatistik der Schweiz. Swiss Federal Statistics Office, Bern, Switzerland. Swisstopo, 2005. Digital elevation model DHM 25. Swiss Federal Office of Topography, Wabern, Switzerland. 
Appendix 2. R-codes for post-processing the outputs of ALUAM-AB

With the code below, Fig. 2, 3 and 4 from the paper were created. The input table for the codes below is "appendix4.csv", which is also supplied in the supplementary materials as an example. This table contains input variables and results from the ALUAM-AB simulations in which direct payments were varied. Some additional processing of the ALUAM-AB output was necessary to create the columns that are based on the linear regression between the time steps and the area of intensive or extensive agriculture (i.e. the columns sigBetaIntensive, sigBetaExtensive, areaIntensive $\mathrm{C} 10 \mathrm{Y}$, areaExtensiveC10Y, betaIntensive and betaExtensive). However, since the output of every agent-based model is formatted differently, it is not possible to provide a generic R-script for these analysis steps. Below is a short description of the different columns in appendix4.csv:

Factor: The multiplication factor with which the direct payments were multiplied relative to the level of direct payments in 2001.

areaIntensive2002: The area of intensive agriculture in 2002 (ha)

areaExtensive2002: The area of extensive agriculture in 2002 (ha)

sigBetaIntensive: The p-value of the regression coefficient of the years regressed against the area of intensive agriculture.

sigBetaExtensive: The $\mathrm{p}$-value of the regression coefficient of the years regressed against the area of extensive agriculture.

areaIntensiveC10Y: The change of the area of intensive agriculture over a 10-year period (ha; betaIntensive * 10).

areaExtensiveC10Y: The change of the area of extensive agriculture over a 10-year period (ha; betaExtensive * 10).

betaIntensive: The regression coefficient of the years regressed against the area of intensive agriculture $\left(\right.$ ha* $^{*}$ year $\left.^{-1}\right)$.

betaExtensive: The regression coefficient of the years regressed against the area of extensive agriculture $\left(\right.$ ha* $^{*}$ year $\left.^{-1}\right)$.

$\underline{\text { Direction field plots }}$

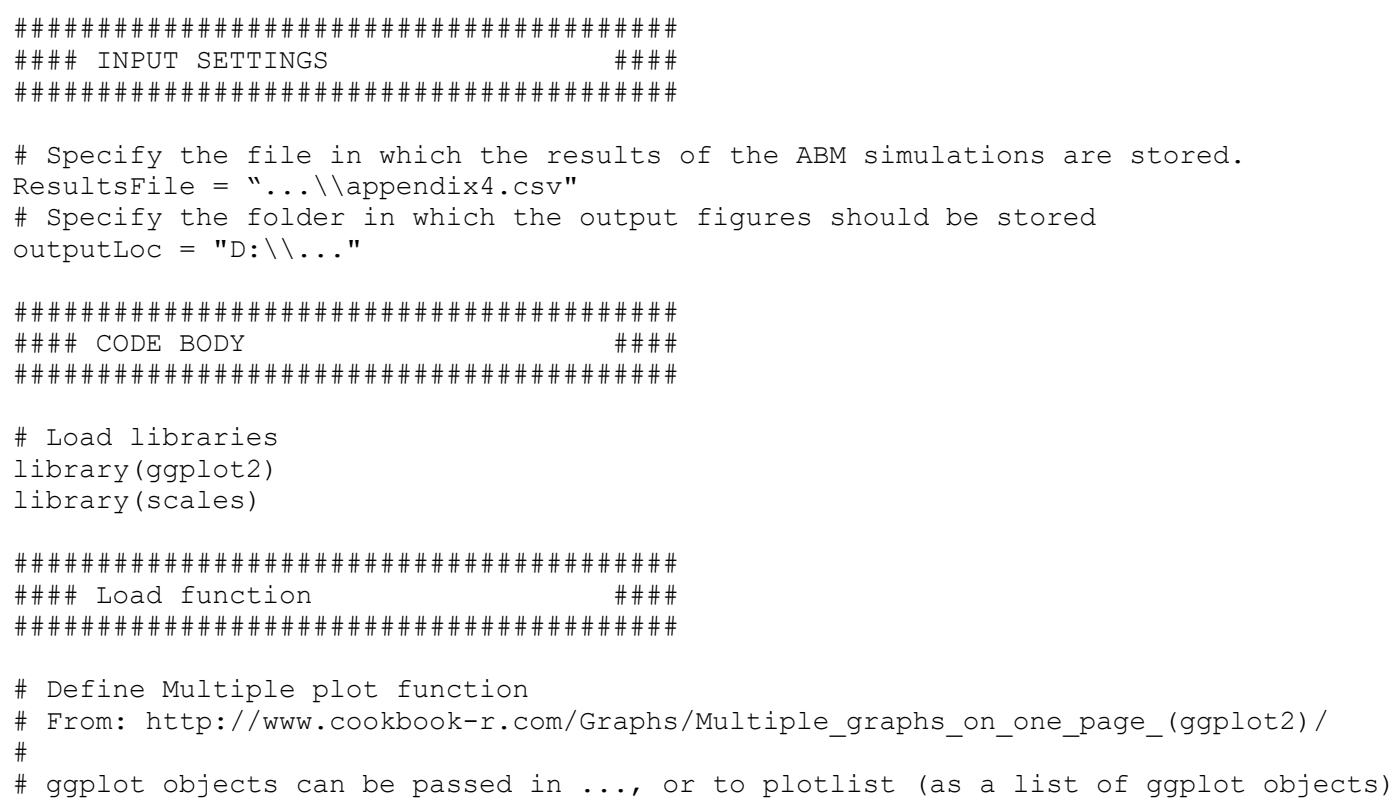




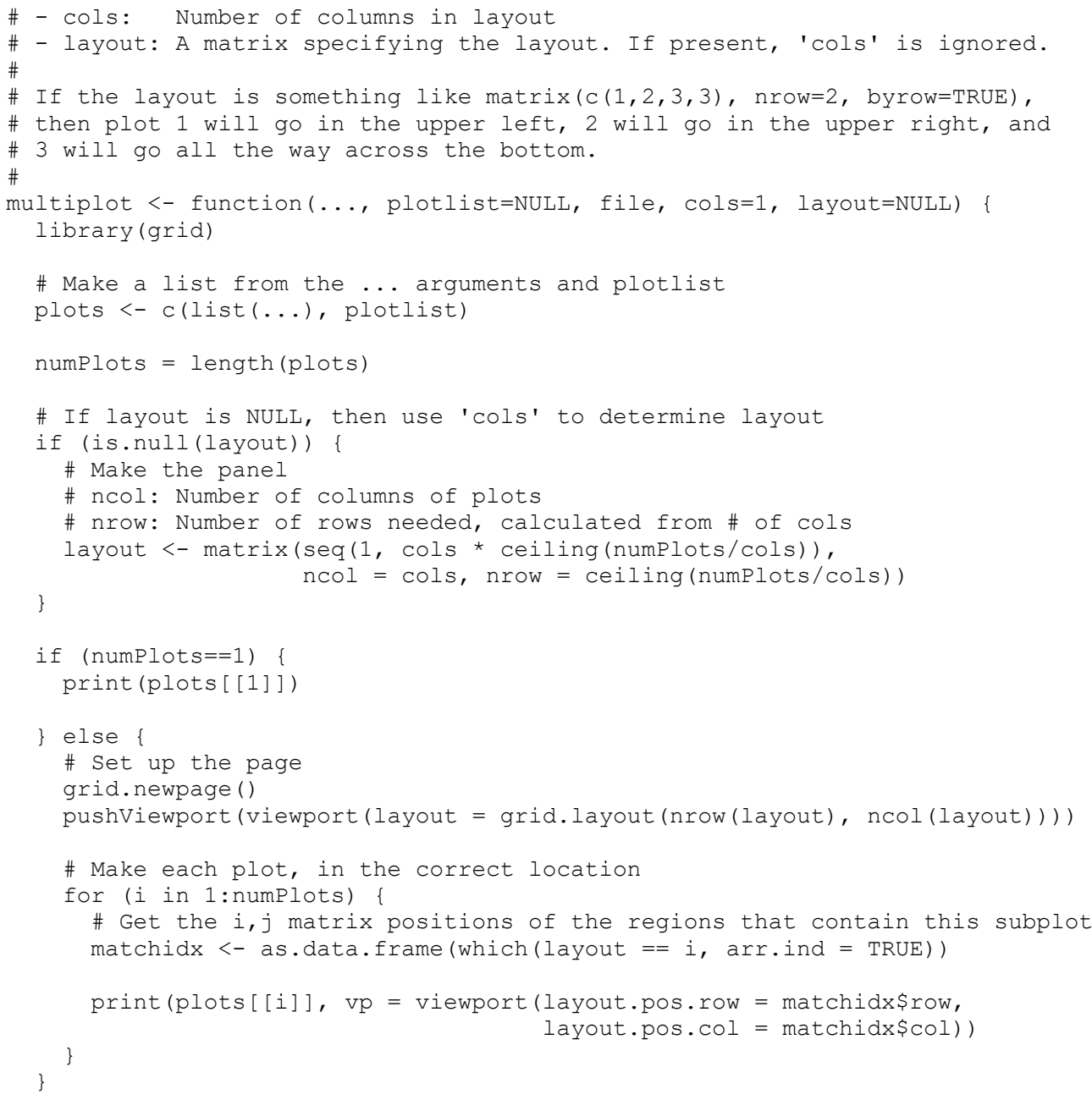

\#Plot the direction field plots for extensive agriculture extensivePlot = ggplot (data=scenarioTabSel, aes (x=Factor, $y=a r e a E x t e n s i v e 2002$, colour $=$ areaExtensivec10Y)) +

geom segment (aes (xend=Factor, yend=areaExtensive2002+areaExtensiveC10Y), arrow =

arrow (length = unit $(0.3, " \mathrm{~cm} ")$, type = "closed"), size = 1.0)+

xlab("Multiplication factor direct payments") +

ylab ("Area extensive agriculture (ha)") +

scale_colour_gradientn(colours=c ("Green","Light green","white","pink","red"), guide = FALSE, values=rescale (c (max (scenarioTabSel\$areaExtensivec10Y), 5, 0,-

5, min (scenarioTabSel\$areaExtensiveC10Y))) ) +

theme_dark ()$+$

theme $\overline{(t e x t=e l e m e n t}$ text $($ size $=25))$

\#Save the plots 
setwd(outputLoc) \#Set the workspace

pdf ("DirectionfieldPlot.pdf",width=20, height=10)

multiplot (intensivePlot, extensivePlot, $\operatorname{cols}=2$ )

dev.off ()

\#Save the plots

setwd(outputLoc) \#Set the workspace

jpeg("DirectionfieldPlot.jpg",width=40, height=20, units = "cm", res=300)

multiplot (intensiveplot, extensivePlot, cols = 2)

dev.off ()

\section{$\underline{\text { Stability landscapes }}$}

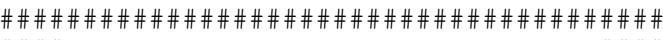

\#\#\#\# INPUT SETTINGS \#\#\#\#

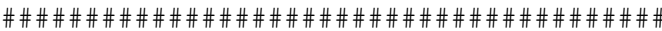

\# Specify the file in which the results of the ABM simulations are stored.

Resultsfile = "... \appendix4.csv"

\# Specify the folder in which the output figures should be stored

outputLoc = "D: \\..."

\# Open an online plotly account. Follow the instructions from here: https://plot.ly/r/gettingstarted/

Sys.setenv("plotly username"="XXXXXXXXXXX") \#Enter plotly user-name

Sys.setenv("plotly api key"="XXXXXXXXXX") \#Enter plotly password

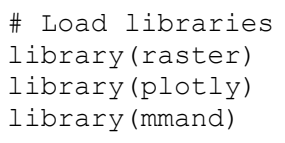

\# Define the function to create the stability landscapes

\# Requires:

\# $\quad \mathrm{x}$ : vector with the values for the x-axis variable (i.e. system stressor)

\# $y$ : vector with the values for the y-axis variable (i.e. system state)

\# beta: vector with the regression coefficient values (i.e. positive or negative growth)

\# size_raster: (optional) the numbers of rows and columns in the grid covering the state space

\# MWsigma: (optional) the sigma parameters used to define the Gaussian weighting kernel

\# xlab: (optional) the label for the x-axis in the 3D stability landscape

\# ylab: (optional) the label for the y-axis in the 3D stability landscape

stabilityLS = function $(x, y$, beta,size_raster=c $(25,25)$, MWsigma=c $(1.5,1.5), x l a b=" x ", y l a b=" y ")$ \{

\# set up an 'empty' raster via an extent object

e <- extent (c(min $(x), \max (x), \min (y), \max (y)))$

$r<-$ raster (e, ncol=size raster [1], nrow=size_raster [2])

\# Convert the beta values to a raster by taking the mean value. This raster will have holes in it.

$\mathrm{x}<-$ rasterize (data.frame $(\mathrm{x}, \mathrm{y}), \mathrm{r}$, beta, fun = mean)

\# Create a Gaussian kernel with sigma $=c(1.5,1.5)$ to use for the focal function below weights = gaussianKernel (sigma = MWsigma)

\# Apply focal statistics with a window of size_window $x$ size_window and apply the mean of this moving window.

$\mathrm{x}$ focal $=$ focal $(\mathrm{x}, \mathrm{w}=$ weights, fun $=$ mean, pad $=$ TRUE, padValue $=$ NA, na.rm=TRUE)

\# Transform the output of the focal to a matrix

$z=\operatorname{as.matrix}\left(x_{-}\right.$focal)

\# Per column calcualte the cumulative sum of the column. 


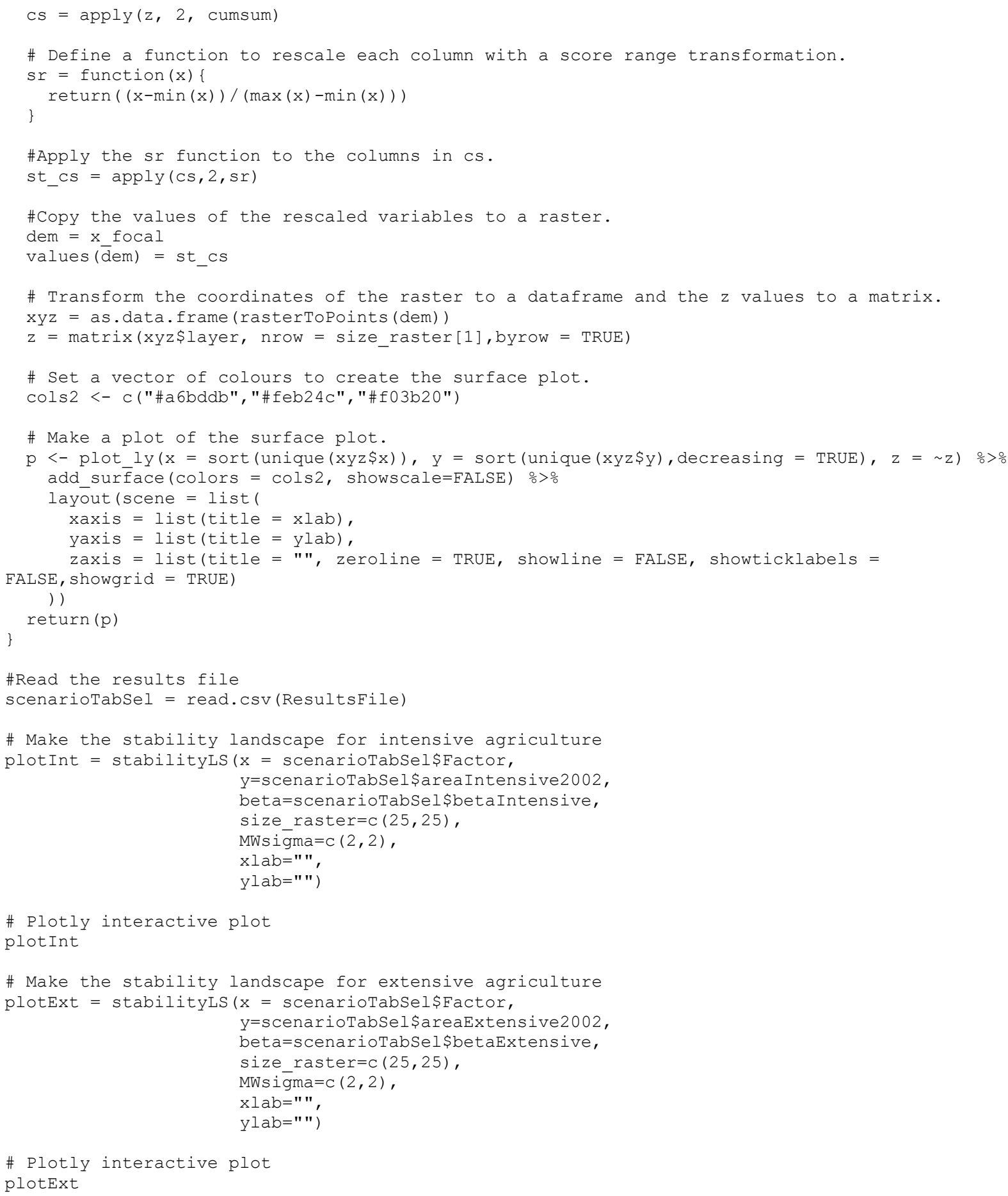

\section{Support vector machine classifications and bifurcation diagrams}

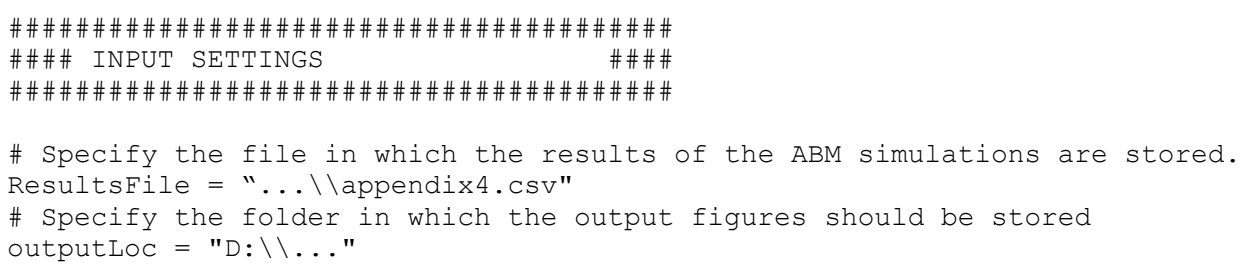




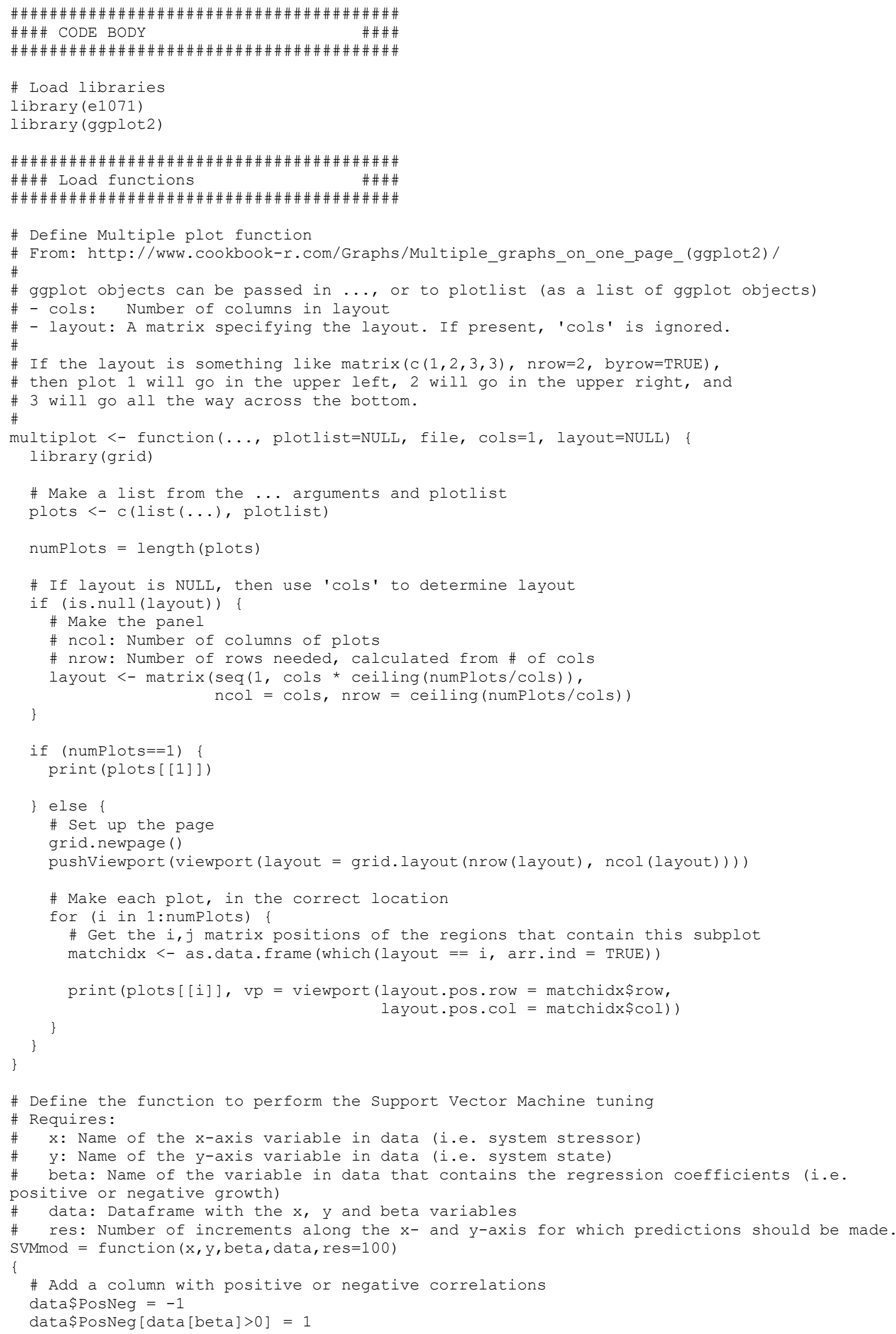




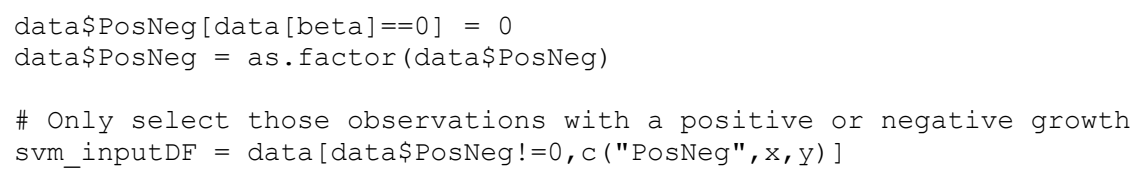


geom_raster (data = IntMod[[3]], aes (x = Factor, $y=$ areaIntensive2002, fill = xy pre $\bar{d})$, alpha=0.1, show.legend $=$ FALSE $)+$

scale_fill_manual (values $=$ colPoly) +

stat_contour (data = IntMod[[3]], aes $(x=$ Factor, $y=$ areaIntensive2002, $z=$ as.numeric(xy pred)), bins $=1$, size $=2$, color = "black") +

xlab("Multiplication factor direct payments")+

ylab("Initial area intensive agriculture (ha)")+

theme (text $=$ element text $($ size=25))

\#Save the plots

setwd(outputLoc) \#Set the workspace

pdf ("SVMplot.pdf", width=20, height=10)

multiplot (IntPlot, ExtPlot, $\operatorname{cols}=2$ )

dev.off ()

\#Save the plots

setwd(outputLoc) \#Set the workspace

jpeg ("SVMplot.jpg", width=40, height=20, units $=" \mathrm{~cm} "$, res=300)

multiplot (IntPlot, ExtPlot, cols = 2)

dev.off ()

\#Print the SVM-classification results for extensive agriculture

print (paste("The best cost hyperparameter for extensive agriculture was:",

ExtMod[[1] ] \$best.parameters\$cost, sep = " "))

print (paste("The best gamma hyperparameter for extensive agriculture was:", ExtMod [ [1]] \$best.parameters\$gamma, sep = " "))

print (paste("The lowest classification error for extensive agriculture was:", ExtMod[[1] ] \$best.performance, sep = " "))

\#Print the SVM-classification results for intensive agriculture

print (paste("The best cost hyperparameter for extensive agriculture was:", IntMod [1] ] \$best.parameters\$cost, sep = " "))

print (paste("The best gamma hyperparameter for extensive agriculture was:" IntMod [ [1]] \$best.parameters\$gamma, sep = " ")

print (paste("The lowest classification error for extensive agriculture was:", IntMod[[1]] \$best.performance, sep = " ")) 
Appendix 3. Additional plots
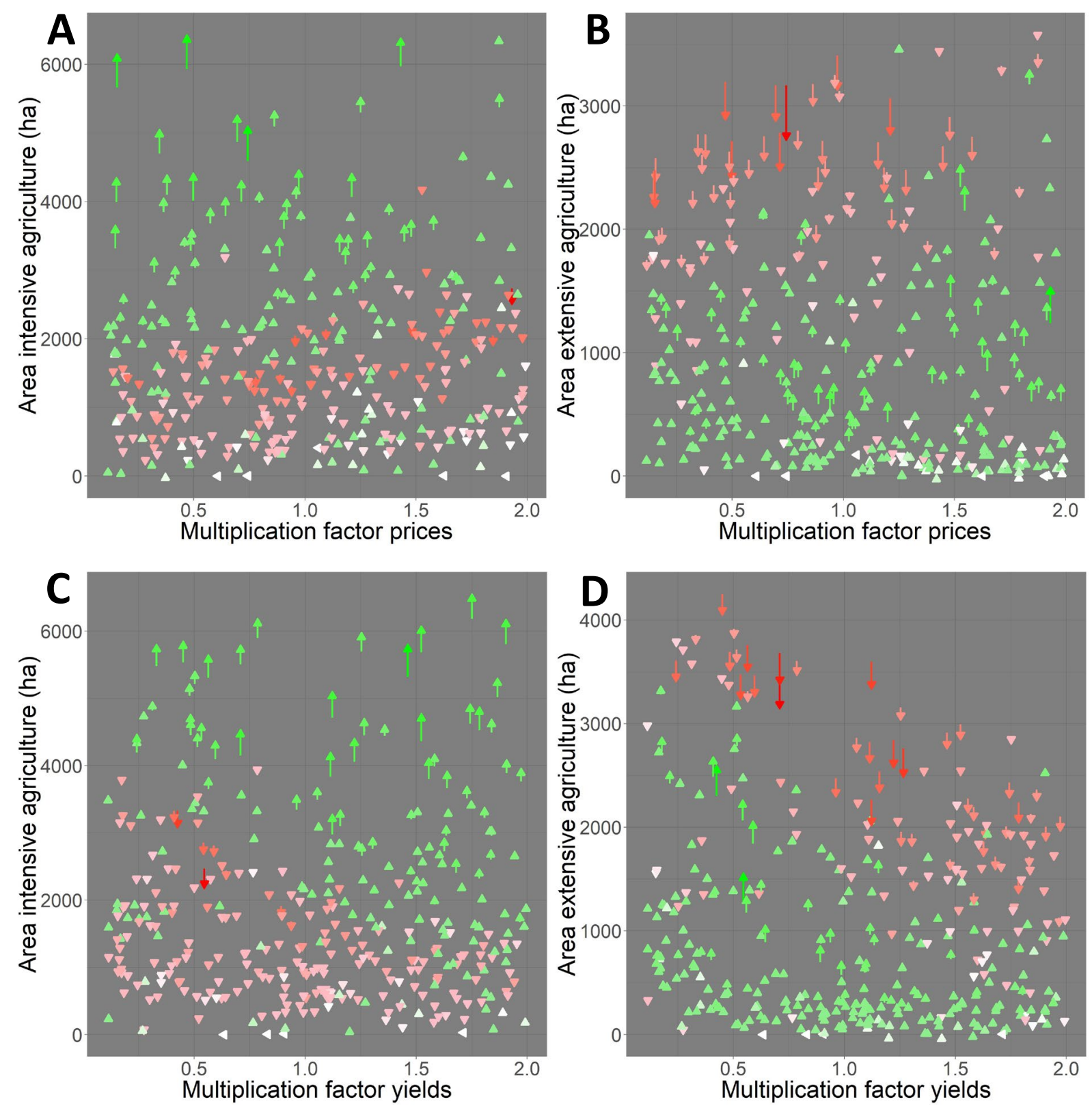

Figure A3.1. Vector-field plots of the direction and magnitude of development of the area of intensive or extensive agriculture under different prices for agricultural commodities (A, B) and different agricultural yields $(\mathrm{C}, \mathrm{D})$. Each arrow (or vector) originates from the point that represents the initial area of intensive $(A, C)$ or extensive $(B, D)$ agriculture and the multiplication factor with which the prices or yields were multiplied (baseline $=1$ ). The arrow represents the 10-year trend for the development of agricultural area. The arrows can indicate a positive (green), negative (red) or no (white) trend. The intensity of the red and green colours increases with an increasing magnitude of change. 

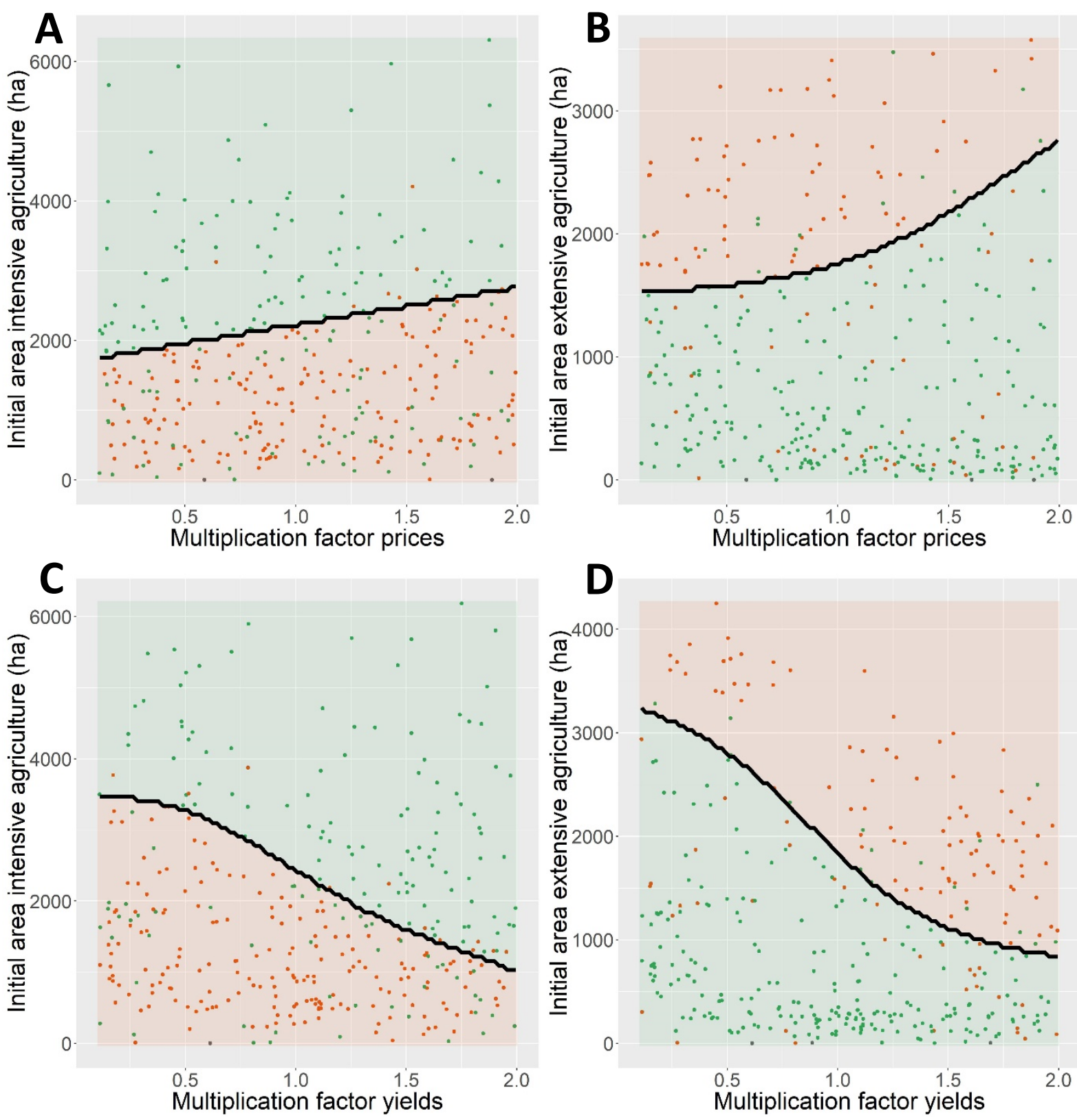

Figure A3.2. Decision surfaces of the support-vector machine classification of areas of positive and negative growth of intensive $(A, C)$ or extensive $(B, D)$ agriculture for different prices for agricultural commodities (A, B) and different agricultural yields $(C, D)$. The points in the plots are the same as the starting points of the arrows in Fig. A3.1. The growth trend in each model run was assigned to either of three classes: positive (green dot), negative (red dot) or flat (grey dot). The positive and negative trends were used to train support-vector machines to find the equilibria (i.e. separator line) between areas of positive (green area) and negative (red areas) growth. In (A, C), the identified equilibrium is unstable, while in $(\mathrm{B}, \mathrm{D})$ the equilibrium is stable. 
Appendix 4. An example input table for the R-codes in Appendix 2. This table contains input variables and results from the ALUAM-AB simulations in which direct payments were varied. Below is a short description of the different columns in appendix4. csv: Factor: The multiplication factor with which the direct payments were multiplied relative to the level of direct payments in 2001. areaIntensive2002: The area of intensive agriculture in 2002 (ha) areaExtensive2002: The area of extensive agriculture in 2002 (ha) sigBetaIntensive: The $\mathrm{p}$-value of the regression coefficient of the years regressed against the area of intensive agriculture. sigBetaExtensive: The p-value of the regression coefficient of the years regressed against the area of extensive agriculture. areaIntensiveC10Y: The change of the area of intensive agriculture over a 10-year period (ha; betaIntensive * 10). areaExtensiveC10Y: The change of the area of extensive agriculture over a 10-year period (ha; betaExtensive * 10). betaIntensive: The regression coefficient of the years regressed against the area of intensive agriculture (ha*year-1). betaExtensive: The regression coefficient of the years regressed against the area of extensive agriculture (ha*year-1). 